\title{
First report of Fusarium proliferatum causing wilt and root rot disease on wild accessions of pigeonpea from India
}

\author{
Raj K. Mishra ${ }^{1} \cdot$ Monika Mishra ${ }^{1} \cdot$ Abhishek Bohra $^{1} \cdot$ Satheesh Naik SJ ${ }^{1} \cdot$ Krishna Kumar $^{1}$
}

Received: 3 April 2021 / Accepted: 4 October 2021 / Published online: 22 October 2021

(c) Società Italiana di Patologia Vegetale (S.I.Pa.V.) 2021

Keyword Fusarium proliferatum $\cdot$ Pigeonpea $\cdot$ Wilt $\cdot$ Molecular identification

Pigeonpea (Cajanus cajan L.) is the sixth most important legume crop in the world. India is the largest producer and consumer. Approximately 15-20\% wild pigeonpea plants from three distinct genera (Cajanus spp., Rhynchosia spp. and Flemingia spp.) in the Research Farm of ICAR-IIPR, Kanpur, India, during 2019-20 were visually affected by wilt and root rot symptoms at flowering and fruiting stage. Initial symptoms of the disease showed chlorosis and drooping of leaves. Infected plants showed browning to blackening discoloration with a gradual drying and eventually death of the plant. Symptomatic plants were sampled from experimental field. Then, $5.0 \mathrm{~mm}$ size pieces of stem tissues surface-sterilized with $1 \%$ sodium hypochlorite for $2 \mathrm{~min}$ were transferred to $90 \mathrm{~mm}$ Petri dishes containing Fusarium Specific Medium (FSM) and incubated at $27^{\circ} \mathrm{C}$. After $48 \mathrm{~h}$ white to light orange mycelial growth was observed. Fungus was further purified by single spore technique. Macro conidia had 3-4 septa, hyaline and slightly curved at apex, ranged from 18.0-22.0 $\times 3.0-5.0 \mu \mathrm{m}$. Microconidia were clavate, hyaline, smooth, $4.0-10.4 \times 2.2-3.1 \mu \mathrm{m}$. No clamydospores were observed. Morphological characteristics of isolates were consistent with the description reported by Leslie and Summerell (2006) of F. proliferatum. Pathogenicity test was conducted on 15-day healthy seedlings of wild pigeonpea (ICP-15882) using 'root dip inoculation' and 'soil inoculation' technique. Disease symptoms observed on inoculated plants after 10 days of inoculation were similar to those observed in fields. The same fungus was re-isolated from the inoculated plants. The identity

Raj K. Mishra

rkmishraiipr@gmail.com

$\triangle$ Monika Mishra

mishramonika27@gmail.com

1 ICAR-Indian Institute of Pulses Research, Kanpur-208024, India of the fungal cultures were confirmed by analyzing the ITS region of rDNA, FUMS gene 1, TEF-1 and Betatubulin gene (Nosratabadi et al. 2018; Stepien et al. 2011). All the sequences, ITS (MG859733, MG859731), Tef1 (MN175178, MN175177), FUMS 1 (MN175180, MN175179) and Beta-tubulin (MK357892, MN205564) showed $99-100 \%$ identity with $F$. proliferatum available at NCBI (KM459007). Phylogenetic analysis clustered isolates readily and consistently with $F$. proliferatum reference strains. To the best of our knowledge, this is the first report of wilt and root rot disease of wild accession of pigeonpea caused by $F$. proliferatum in India.

Acknowledgements Authors greatly acknowledge the Indian Council of Agricultural Research (ICAR), India for financial support.

\section{Declarations}

Conflict of interest Authors declare that they have no conflict of interest.

\section{References}

Leslie JF, Summerell BA (2006) The Fusarium Laboratory Manual. Blackwell Publishing, Oxford, UK

Nosratabadi M, Kachuei R, Rezaie S et al (2018) Beta-tubulin gene in the differentiation of Fusarium species by PCR-RFLP analysis. Le Infezioni in Medicin 1:52-60

Stępień Ł, Koczyk G, Waśkiewicz A (2011) FUM cluster divergence in fumonisins-producing Fusarium species. Fungal Biol 115:112-123

Publisher's Note Springer Nature remains neutral with regard to jurisdictional claims in published maps and institutional affiliations. 